EPJ Web of Conferences 41, 05041 (2013)

DOI: $10.1051 /$ epjconf/20134105041

(C) Owned by the authors, published by EDP Sciences, 2013

\title{
Model-free Investigation of Ultrafast Bimolecular Chemical Reactions: Bimolecular Photo Induced Electron Transfer
}

\author{
Bernhard Lang, Arnulf Rosspeintner, and Eric Vauthey \\ University of Geneva, Department of Physical Chemistry, Switzerland
}

\begin{abstract}
Using photoinduced bimolecular electron transfer reactions as example we demonstrate how diffusion controlled bimolecular chemical reactions can be studied in a model-free manner by quantitatively combining different ultrafast spectroscopical tools.
\end{abstract}

\section{Introduction}

Bimolecular electron transfer (ET) is often considered as the most simple bimolecular chemical reaction. Nevertheless, many important pieces to a fundamental understanding are yet missing [1], amongst them such crucial points as the distance at which ET actually takes place and the mutual orientation of the reaction partners at that instant. Even the degree to which the latter may determine reaction rates and kinetics is not known so far. Yet, such information would be of utmost importance for a fundamental understanding of bimolecular chemical reaction dynamics and control, especially once a reaction takes place on the ultrafast time scale and beyond pure thermodynamic control. Besides the complication of diffusion control, several technical difficulties account for the apparent lack of information. In the visible spectral domain, the signatures of the various states involved in the course of a chemical reaction overlap in time and spectrum. Furthermore, processes inevitably accompanying a photochemical reaction on the time scale of the ongoing reaction itself, like vibrational cooling and solvent relaxation, add further shifting components to the transient spectra, respectively significantly alter the shapes of the signatures of interest.

Decomposition techniques like target analysis together with global fitting and singular value decomposition have been successfully applied to disentangle the various compounds of transient spectra [2]. However, a successful application of such methods is bound to the existence of a reliable kinetic model. Furthermore, components with shifting or changing shapes are difficult to cope with using such techniques. The reliability of the findings will always depend on the reliability of the employed model, with the risk-in worst case-to obtain as a result what has been implicitly imposed by the kinetic model.

In this contribution we show how the combination of different ultrafast spectroscopic methods can circumvent the problem of a-priory needed substantial information on the reaction kinetics. In the example demonstrated in the following we have been able to track the pathway of an ultrafast bimolecular charge separation (CS) and recombination (CR) reaction by extracting hot ground state spectral signatures where neither their shape nor any of the involved kinetics (being highly non-exponential) were known a-priori.

\section{Experiment}

It is one of the long lasting questions around bimolecular CS whether a radical ion pair could be formed with one of the ions initially being in an electronically excited state. Figure 1 shows the energy diagram of a bimolecular CS and CR reaction with two donor acceptor (DA) pairs, perylene (Pe) with tetracyanoethylene (TCNE) on the left and cyanoperylene (CNPe) with TCNE on the right panel.

This is an Open Access article distributed under the terms of the Creative Commons Attribution License 2.0, which permits unrestricted use, distribution, and reproduction in any medium, provided the original work is properly cited. 

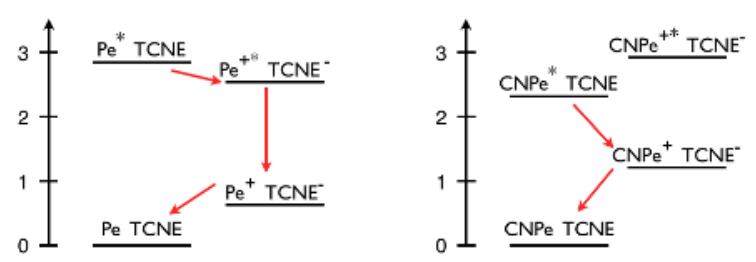

Fig. 1. Energy diagram for electron transfer and recombination on two donor-acceptor pairs.

With $\mathrm{Pe}+\mathrm{TCNE}$, the series of $\mathrm{D}$ states of the $\mathrm{Pe}^{\bullet+}$ radical cation is energetically accessible when photoexciting Pe to its $\mathrm{S}_{1}$ state. With CNPe and TCNE on the other hand, no such state is accessible. A direct spectroscopic approach to address the question whether the excited state of the Pe radical cation can get populated during CS is practically impossible since the excited states of such radical ions are in general very short lived.

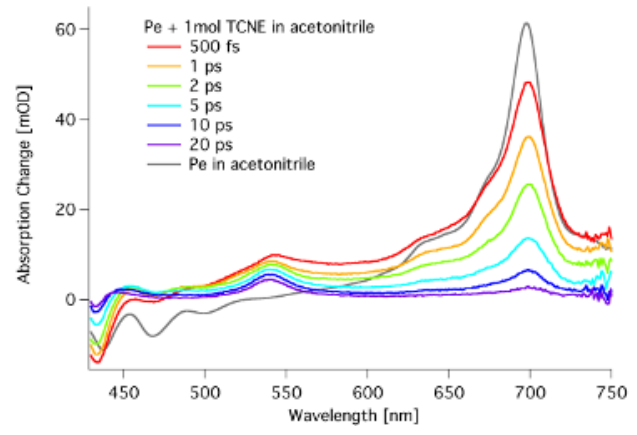

Fig. 2. Transient absorption spectra of Pe upon photoinduced electron transfer with TCNE.

Our approach to this question makes use of the fact that when forming $\mathrm{Pe}^{\bullet+}$ in an excited state, the subsequent relaxation to its ionic ground state by ultrafast internal conversion will release the corresponding energy uniquely to the $\mathrm{Pe}^{\bullet+}$ molecule. In the case where no electronic excited state is accessible, the whole energy can be distributed more or less symmetrically on both reaction partners, with the partitioning depending on the density of accessible vibrational states. Figure 2 shows a series of transient absorption spectra of Pe and TCNE in acetonitrile (ACN). The gray curve shows a transient absorption spectrum of $\mathrm{Pe}$ in $\mathrm{ACN}$ without quencher for comparison. The apparent features are the broad $\mathrm{Pe} \mathrm{S}_{1}$ excited state absorption with its maximum at $700 \mathrm{~nm}$, the stimulated Pe emission between $450 \mathrm{~nm}$ and $550 \mathrm{~nm}$, the Pe ground state bleach at the blue end of the observation window and the $\mathrm{D}_{0}-\mathrm{D}_{5}$ absorption band of the $\mathrm{Pe}^{\bullet+}$ radical cation at $540 \mathrm{~nm}$. The decay of the $\mathrm{S}_{1}$ band indicates a complete quenching of the $\mathrm{Pe} \mathrm{S}_{1}$ state within 50ps by CS. The cation band rises within time resolution of the experiment and drops to a level which remains constant on our observation window of $2 \mathrm{~ns}$, displaying the fraction of the ion population which eventually separates into free ions. Note that the lowest excitation of the $\mathrm{TCNE}^{\bullet-}$ radical anion starts below $400 \mathrm{~nm}$ so that it does not contribute to the spectral range shown here. The broad and featureless absorption between $450 \mathrm{~nm}$ and $650 \mathrm{~nm}$ at early times (c.f. the curve in gray) can be attributed to the absorption of the vibrationally hot neutral Pe ground state upon CS and CR. A straight forward decomposition of these spectra is not possible, since not only the broad contribution of interest alters rapidly its shape; also the other components exhibit to some extent spectral dynamics. Though being small compared to their absolute signal intensity, they sum up to errors as large as the intensity of the hot ground state signal. To conclude, it is in general very difficult to extract at the same time kinetics and spectral shapes from a single set of measurements, even in a case like the present where the contributions are located at quite different spectral positions with only moderate overlap. However, a decomposition is possible when the isolated spectra and kinetics can be obtained from different but quantitatively matched experiments. 

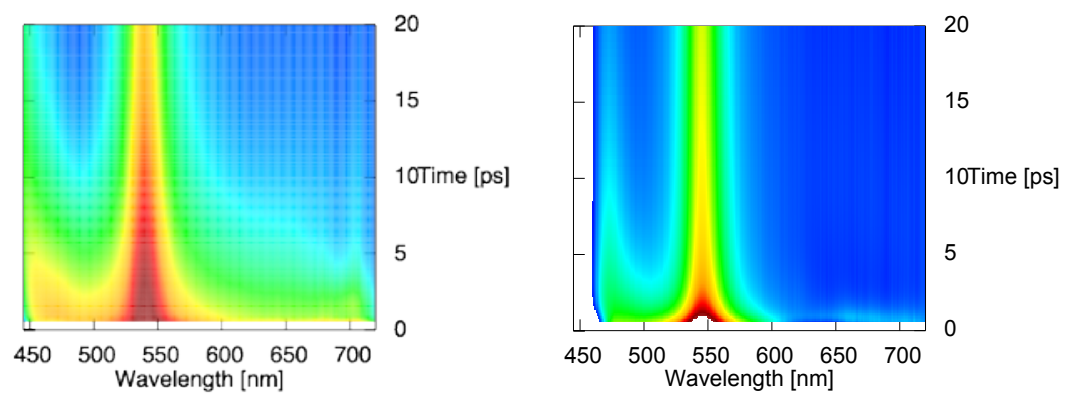

Fig. 3. Hot ground state absorption and ion population of Pe (left) and CNPe (right).

FIG. 3, left panel, shows the isolated contribution of the $\mathrm{Pe}^{\bullet+}$ radical cation and the hot ground state signal upon ultrafast ET to TCNE and subsequent CR. Shape and spectral dynamics of the Pe $\mathrm{S}_{1}$ excited state absorption and stimulated emision have been measured under identical experimental conditions on a sample with Pe in ACN without presence of TCNE. The $S_{1}$ kinetics under presence of TCNE have been determined in the same sample as used for the transient absorption experiment, measuring time resolved fluorescence up-conversion at a carefully chosen emission wavelength where the spectral dynamics cancel out. These kinetics are used to scale the separately measured pure $\mathrm{S}_{1}$ signature accordingly. The result is subtracted from the transient absorption spectra obtained in presence of TCNE. By adjusting a single calibration constant which accounts for the different detection efficiencies in the two experiments, the Pe $S_{1}$ contribution could be removed within an error of 2-3\%. Employing multi-exponential algebraic global analysis [3] with sufficient components so that the residuals have essentially the character of white noise further improves the quality of the decomposition.

During the first picoseconds after photoexcitation of Pe there is a strong signal to be seen which we attribute to the hot Pe ground state. The identical experiment on the pair CNPe + TCNE (fig. 3, right panel) reveals a much less pronounced hot ground state signature. We can conclude that indeed a large amount of heat release to the Pe ground state is found where a formation of the $\mathrm{Pe}^{\bullet+}$ radical cation in an excited electronic states is possible from an energetic point of view. The heat release is largely reduced on the other hand, when such pathway is not accessible.

Measurements on other DA pairs we have performed follow the same line. Furthermore, in a previous study using VIS-IR transient absorption we could determine the heat release on the TCNE side, monitoring the ground state bleach of the antisymmetric CN stretch vibration of TCNE with different donor molecules [4]. All pairs so far investigated in the visible and infrared spectral domain follow the same trend.

\section{Conclusions}

In this contribution we have shown how to track the pathway of a bimolecular reaction under diffusion control by combining different ultrafast spectroscopic tools. Though considered to be standard methods, achieving the neccessary quantitative photometric resolution still asks for considerable efford. Such a combination permits a model-free decomposition of transient absorption spectra and extraction of components where neither shape nor kinetics are a-priori known or can be guessed from the raw data.

\section{References}

1. E. Vauthey, Photochem. Photobiol. A 179, 1 (2006).

2. I. H. M. van Stokkum et al. Biochim. Biophys. Acta 1657, p. 82-104 (2004).

3. P. Fita et al. J. Chem. Phys. 125, 184508 (2006).

4. O.F. Mohammed et al. Angew. Chem. Int. Ed., 47 (47), 9044-9048 (2008). 